\title{
Electron Cyclotron collisionless interaction during EC-assisted tokamak start-up
}

\author{
Daniela Farina ${ }^{1, *}$ \\ ${ }^{1}$ Istituto di Fisica del Plasma, CNR, via Cozzi 53, 20125 Milano, Italy
}

\begin{abstract}
.
In the initial phase of a plasma discharge with EC-assisted breakdown, the wave-particle interaction is nonlinear and wave trapping provides the mechanism for transition from a very low energy state to a much larger energy state. A Hamiltonian adiabatic approach provides the condition for the energy variation to occur in a rigorous way, together with quantitative estimate as a function of the wave frequency, harmonic number, polarisation and EC power and beam width, for the first, and second cyclotron harmonic. The interaction is highly localized in space close to the EC resonance. The spatial profile of the energy gain are reported together with the estimates of the width of the radial region where the interaction takes place in the case of a tokamak configuration. The analysis provides a physics based description of the process as well as indications for an optimized experimental setup.
\end{abstract}

\section{Introduction}

In ITER it is foreseen to startup the plasma injecting Electron Cyclotron (EC) waves to reduce the volt-second consumption of the main transformer during breakdown. This method is used routinely in stellarators as well as in other tokamaks.

Various physical mechanisms occur during r.f. assisted plasma startup, like wave-particle interaction, particle collisions with both neutrals and ionised plasma, particle confinement and plasma build-up, and the overall process is quite complex. The analysis presented here is focussed on the very first phase of the process, i.e., on the EC wave interaction with the seed electrons in the vacuum chamber, which takes place before collisions start to play a role. The electron dynamics under the action of a localised electromagnetic (e.m.) field is investigated theoretically aiming to determine conditions and parameters for which the electrons can achieve enough energy to start the ionisation process. A detailed investigation of this problem has been reported recently in $[1,2]$. Here, the theoretical analysis is summarised for sake of completeness and some new results are presented which are of practical interest to design the experimental setup.

During the initial phase of the EC-assisted breakdown, electrons are assumed to behave as free particles at room temperature moving along the static magnetic field, under the action of a localised microwave beam. As it was first pointed out long ago in [3], such cold electrons can acquire a large energy via nonlinear wave trapping for beam power values like those injected in fusion machines $(100 \mathrm{~kW}-1$ MW in single rf source), whenever the e.m. amplitude is large enough and/or the time of flight in the EC beam is long enough to exceed the nonlinear trapping period.

The wave particle nonlinear interaction process is conveniently investigated within a Hamiltonian adiabatic formalism, following the theoretical approach developed in [3-9] and adopted in [1, 2], which allows the derivation of the conditions at which the particles gain energy in single beam crossing in a rigorous way and the quantitative dependence of the energy variation on wave frequency, harmonic number, polarisation and EC power and beam width. The analysis applies to timescales satisfying the following conditions

$$
\tau_{\text {cycl }} \ll \tau_{\text {trap }} \ll \tau_{\text {flight }} \ll \tau_{\text {coll }},
$$

being the cyclotron period, the trapping time in the wave field, the time flight in the beam and the collision time, respectively. In ITER typical parameters are $\tau_{\text {cycl }} \simeq 6 \times$ $10^{-12} \mathrm{~s}, \tau_{\text {trap }} \approx 10^{-8} \mathrm{~s}, \tau_{\text {flight }} \approx 1-5 \times 10^{-6} \mathrm{~s}, \tau_{\text {coll }} \approx$ $5 \times 10^{-4}-5 \times 10^{-3} \mathrm{~s}$, Equation (1) identifies a nonlinear adabiatic interaction regime.

\section{Nonlinear interaction}

In a tokamak, EC assisted startup occurs in the magnetic null region close to the EC cold resonance.

The electron motion can be modelled in a simple way as that of a free charged particle in a given static, uniform and constant magnetic field $\mathbf{B}_{0}=B_{0} \mathbf{e}_{z}$ (representing the vacuum toroidal field), interacting with a localised e.m. beam with frequency $\omega$ close to a harmonic of the cyclotron frequency $\Omega=\left|q_{e}\right| B_{0} / \mathrm{m}$. The motion equations read

$$
\frac{d \mathbf{x}}{d t}=\mathbf{v}, \quad \frac{d \mathbf{p}}{d t}=q_{e} \mathbf{E}(\mathbf{x}, t)+q_{e} \mathbf{v} \times\left[\mathbf{B}_{0}+\mathbf{B}(\mathbf{x}, t)\right],
$$


where $\mathbf{p}=m \gamma \mathbf{v}$ is the kinetic momentum, $\mathbf{v}$ the electron velocity, $\gamma=\sqrt{1+p^{2} / m^{2} c^{2}}$ the relativistic factor, $\mathbf{x}$ the coordinate vector, $q_{e}$ the (negative) electron charge and $\mathbf{E}(\mathbf{x}, t)$ and $\mathbf{B}(\mathbf{x}, t)$ the electric and magnetic field of the wave, with $\mathbf{E}(\mathbf{x}, t)=E\left[e_{x} \sin \chi \hat{\mathbf{x}}-e_{y} \cos \chi \hat{\mathbf{y}}+e_{z} \sin \chi \hat{\mathbf{z}}\right]$, $\chi=k_{\perp} x+k_{\|} z-\omega t$ the wave phase, $\mathbf{e}=\mathbf{E} /|\mathbf{E}|$ the wave polarisation vector, and $k_{\perp, \|}$ the wave vector components perpendicular and parallel to the static magnetic field. The EC beam is assumed to have a Gaussian-like profile with wave electric field amplitude $E=E_{M} \exp \left(-r^{2} / w^{2}\right)$, being $E_{M}=2\left[\eta_{0} P /\left(\pi w^{2}\right)\right]^{1 / 2}$ its maximum value (in SI units), $r$ the beam radius, $w$ the local beam width, $P$ the incident EC power and $\eta_{0} \simeq 376.73 \mathrm{ohm}$ the free space impedance.

In the Coulomb gauge with zero scalar potential, the electric $\mathbf{E}$ and magnetic $\mathbf{B}$ fields are related to the vector potential $\mathbf{A}(\mathbf{x}, t)=\mathbf{A}_{0}(\mathbf{x})+\mathbf{A}_{1}(\mathbf{x}, t)$ as $\mathbf{E}=-\partial \mathbf{A} / \partial t$, and $\mathbf{B}=\nabla \times \mathbf{A}$, and the electron motion is described by the Hamiltonian function

$$
H(\mathbf{x}, \mathbf{P})=m c^{2} \gamma=m c^{2}\left(1+p^{2} / m^{2} c^{2}\right)^{1 / 2},
$$

being $\mathbf{P}=\mathbf{p}+q_{e} \mathbf{A}$ the canonical momentum conjugate to $\mathbf{x}$. Dimensionless quantities and variables are used, with time, length, momentum, energy and Hamiltonian, and vector potential normalised over $\Omega^{-1}, c \Omega^{-1}, m c, m c^{2}$, and $\left|q_{e}\right| / m c$, respectively.

Following the approach described e.g. in [8], the Hamiltonian transforms via canonical transformations to a time-independent Hamiltonian function $H$ with two degrees of freedom, valid for $\omega$ close to a cyclotron harmonic $n \Omega$. At first order in the normalised wave amplitude $\epsilon(z)=\left|q_{e}\right| E(z) / m c \omega \ll 1$, it reads

$$
H\left(z, \theta, \bar{P}_{z}, I\right)=\gamma-v_{n} I=H_{0}\left(\bar{P}_{z}, I\right)+\epsilon(z) H_{1}\left(z, \theta, \bar{P}_{z}, I\right)
$$

where $v_{n}=v / n=\omega / n \Omega, H_{0}\left(\bar{P}_{z}, I\right)=\Gamma-v_{n} I$, $H_{1}\left(z, \theta, \bar{P}_{z}, I\right)=\Theta_{n}\left(\bar{P}_{z}, I\right) / \Gamma \cos n \theta$, with $I$ and $\theta$ actionangle variables, $\bar{P}_{z}=P_{z}-N_{\|} v_{n} I$ the canonical momentum conjugate to $z$, and $\Gamma=\left(1+2 I+P_{z}^{2}\right)^{1 / 2}$. For the unperturbed system, $\epsilon(z)=0, \theta$ is the phase of the gyromotion, $I=p_{\perp}^{2} / 2$, and $P_{z}=p_{z}$. The polarisation term $\Theta_{n}$ reads $\Theta_{n}=\sqrt{2 I}\left[e_{-} J_{n-1}(b)+e_{+} J_{n+1}(b)\right]+P_{z} e_{z} J_{n}(b)$ with $e_{ \pm}=\left(e_{x} \pm e_{y}\right) / 2$ and $J_{n}$ the Bessel function of argument $b=v N_{\perp} \sqrt{2 I}$. Hereafter, two normal modes in vacuum are considered with the same polarization as the Ordinary (OM) and eXtraordinary (XM) modes of cold plasmas in the limit of zero density.

Due to the constancy of the Hamiltonian, the following relationships hold between the initial and final electron states

$$
\begin{aligned}
\Delta \gamma & =v_{n} \Delta I \equiv v_{n} \Delta\left(p_{\perp}^{2} / 2\right), \\
\Delta p_{z}^{2} & =\Delta \gamma\left[\Delta \gamma-2\left(1 / v_{n}-\gamma_{0}\right)\right],
\end{aligned}
$$

so that the computation of $\Delta I$ provides both the total and the parallel final energy. The first relation holds also at any time, $\delta \gamma=v_{n} \delta I$, and in addition one has $\delta P_{z}=\delta \bar{P}_{z}+$ $N_{\|} v_{n} \delta I$. Outside the beam region, the parallel canonical momentum $\bar{P}_{z}$ is a constant of motion.

The main features of the interaction process can be studied solving the Hamiltonian equation of motion for thermal electrons at room temperature $T_{e}=0.03 \mathrm{eV}$ with initial conditions outside the EC beam illumination region. In Figure 1 the behaviour of the kinetic energy $W=m c^{2}(\gamma-1)$ versus the spatial coordinate $z$ is shown for two electrons with same initial conditions except for the initial phase value $\theta$, for second harmonic, XM polarised beam in oblique propagation. While the electrons cross the EC beam, they become non linearly trapped in the wave field, experience large energy variations with maximum value at $z=0$ where the wave field amplitude is maximum. When the e.m. field amplitude decreases $(z>0)$ the electrons are detrapped for the same $E$ value, and exit the beam region with final energy either quite large (Figure 1a) or equal to the initial one (Figure 1b).

The numerical investigation of the electron motion reported in [1] showed that a cold electron may gain a significant amount of energy when it flows along a magnetic field line under the action of a localised EC beam at frequency close to the first and second cyclotron harmonics. Under adiabatic conditions, the energy that a cold electron gains after beam crossing is quite large and independent of the beam power and width, provided that the electron is non linearly trapped in the wave field. At fixed beam parameters, the gained energy increases with the frequency mismatch from the resonance up to a critical value beyond which is zero. Trapping occurs if the maximum electric field amplitude exceeds a threshold value that depends on the harmonic number, wave frequency, injection angle and mode polarisation.

Whenever the flight time in the beam region is larger than the trapping time in the wave field and the e.m. amplitude variation is sufficiently slow, the Hamiltonian is characterised by a couple of fast action-angle variables $(\theta, I)$ and a couple of slow canonically conjugate variables $\left(z, \bar{P}_{z}\right)$, and the action integral $J\left(z, \bar{P}_{z}\right) \equiv n / 2 \pi \oint I d \theta$ is an adiabatic invariant of the motion, that breaks only at the separatrix crossing between trapped and untrapped orbits. Breaking of the invariant via trapping/untrapping allows to connect different regions in phase space and provides the mechanism for net energy variation to occur. This mechanism for EC nonlinear interaction is the core physical phenomenon [3-9].

A few critical conditions need to be fulfilled in order to give rise to adiabatic nonlinear energy gain: i) the initial status, namely the electron parallel momentum, the frequency ratio $v_{n}$ and the local $N_{\|}$, correspond to a "resonant case" for the unperturbed motion, such that two different energy states (low and high respectively) exist for the same value of the constant of motion $H$; ii) connection between these two energy states occurs via nonlinear trapping, which implies a threshold condition on the wave amplitude; iii) in addition, at trapping/detrapping the separatrix splits the phase space of the fast variables in three regions, corresponding to the trapped motion, and to the two free streaming motion at different energy levels.

The process can be sketched in the phase space of the fast variables $(I, \theta)$ (see e.g. Figure 1 in [4] and Figure 4 in [2]), whenever a resonant condition is satisfied and the Hamiltonian is double-valued. Outside the beam region the electron lies on a given branch of the Hamiltonian $H_{0}$. 
When it flies in the illumination region along the magnetic field line, it experiences an increasing field amplitude $\epsilon$ and is eventually trapped in the wave field at some $\epsilon_{s}$ value if lower than the maximum value $\epsilon_{M}$. The lower and upper branches of the Hamiltonian corresponding to same value merge together at the separatrix crossing. For decreasing $\epsilon$ values detrapping occurs at $\epsilon_{s}$ and the particle may leave the separatrix either on the upper or lower branch, so that after beam crossing both branches can be populated. A net energy variation occurs with a given probability only if after the last separatrix crossing the particle will lie on the other branch of the Hamiltonian.

This process is illustrated in Figure 2 where the results of the same simulations shown in Figure 1 are reported in the "phase" space $W, \theta$ (note that $W$ is proportional to the action $I$ conjugate to $\theta$ ). Initially the particle follows a trajectory quite close to the horizontal axis and, after crossing of the separatrix, it oscillates around the elliptic point at $\theta=0$. When the electric field amplitude decreases the particle is untrapped, and two different situations can occur depending on the Hamiltonian branch on which the particle lies. After exiting the illumination region, the particle describes a horizontal line in phase space either (a) at large energy or (b) at very low energy (note that the maximum energy is $\approx 7 \times 10^{3}$ its initial value so that the details of the motion at low energy are barely seen when linear scale is used). The structure of the phase space $(I, \theta)$ for the first three harmonics is not discussed in detail here and can be found in [1] for the first three harmonics.

An example of motion for which nonlinear wave trapping does not occur is shown in Figure 3, which refers to the same parameters as in Figures 1, and 2, but at lower power. A similar behavior is observed increasing the frequency mismatch from the resonance at fixed power. After crossing the EC beam the electron recovers exactly the same energy as initially.

The above mechanism can be described formally within the Hamiltonian formalism, making use of the constancy of the Hamiltonian and of the adiabatic invariant in each stage of the motion, i.e., $J\left(\epsilon, \bar{P}_{z}\right)=I_{0}, I_{f}$ in the initial and final phases respectively, in which electrons are not trapped (for $\epsilon \leq \epsilon_{s}$ ), and $J\left(\epsilon, \bar{P}_{z}\right)=J_{s}=A_{s} / 2 \pi$ in the trapped phase (for $\epsilon_{s} \leq \epsilon \leq \epsilon_{M}$ ), where $A_{s}$ is the area of the phase space region inside the separatrix at its crossing. If trapping does not occur, $J\left(\epsilon, \bar{P}_{z}\right)=I_{0}$ at any $\epsilon$. The total variation of the action variable $I$ is equal to the jump of the adiabatic integral at the separatrix

$$
\Delta \gamma=v_{n}\left(I_{f}-I_{0}\right)=v_{n}\left(\left|J_{f}\left(\epsilon_{s}, \bar{P}_{z s}\right)\right|-\left|J_{0}\left(\epsilon_{s}, \bar{P}_{z s}\right)\right|\right)
$$

where $\bar{P}_{z s}$ is the $\bar{P}_{z}$ value at the separatrix, and $J_{0, f}\left(\epsilon, \bar{P}_{z}\right)$ is the action integral before and after the separatrix crossings. The $\epsilon$ and $\bar{P}_{z}$ values at the separatrix are related to the initial (or final) momentum values by means of the following system of equations

$$
\begin{gathered}
I_{0, f}=\left|J_{0, f}\left(\epsilon_{s}, \bar{P}_{z s}\right)\right|, \\
H_{0}\left(I_{0, f}, \bar{P}_{z 0, f}\right)=H_{s}\left(\epsilon_{s}, \bar{P}_{z s}\right),
\end{gathered}
$$

where $H_{S}$ is the Hamiltonian function at the separatrix.
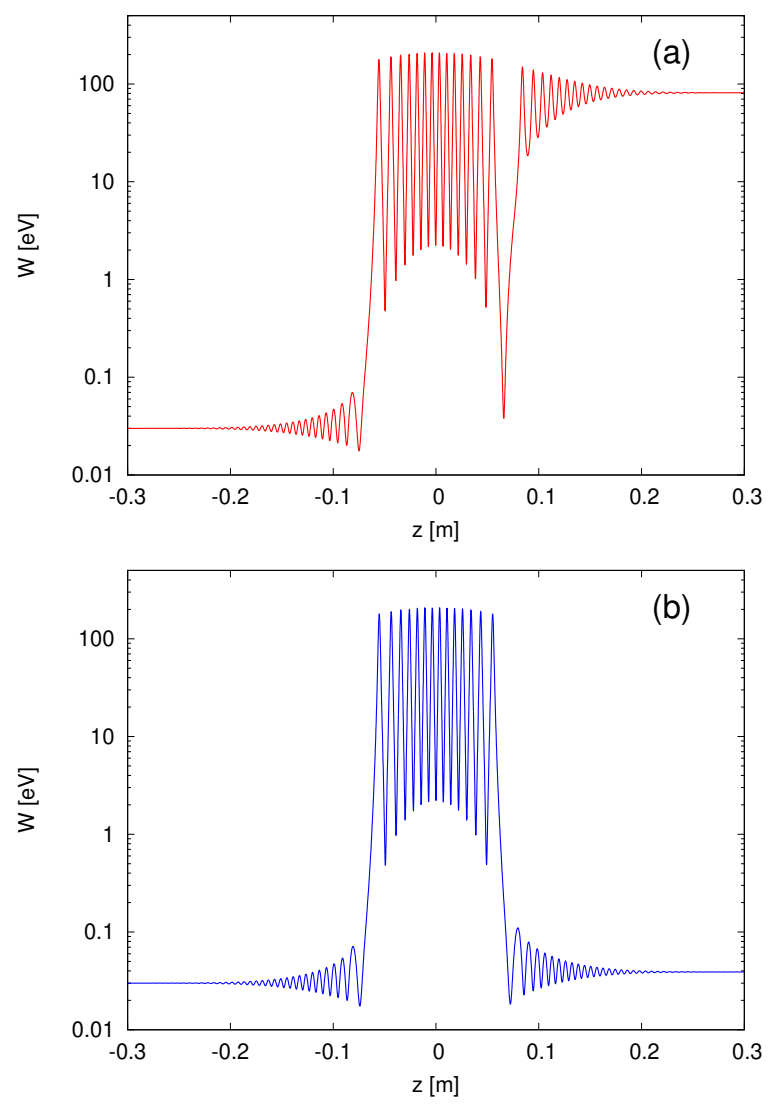

Figure 1. Electron energy $W=m c^{2}(\gamma-1)$ versus spatial coordinate $z$, obtained solving the Hamiltonian equations of motion for two different initial phase values $\chi$, (a) and (b), respectively). The chosen parameters are: EC power $P=1 \mathrm{MW}$, beam width $w=10 \mathrm{~cm}$, frequency ratio $2 \Omega / \omega=1$, for second harmonic XM polarisation in oblique propagation with $N_{\|}=0.3$.

Although the physical process has been reduced to the solution of system of two equations only (7), it is still not straightforward to solve in general. However, in the case under investigation analytical solutions can be found [1] making simplifying approximations that are well justified for the case of very cold electrons: i) weakly relativistic approximation, ii) negligible initial energy, iii) canonical momentum $\bar{P}_{z}$ almost constant for most cases.

As demonstrated in [1], the nonlinear interaction is effective only for the first and the second cyclotron harmonics, this fact being related to the specific topology of the phase space. In fact the phase space of the fast variables $\theta, I$ evolves for increasing perturbation parameter $\epsilon$ from a pendulum-like structure to a more complicate structure when finally the elliptic and hyperbolic points merge together at a critical value $\epsilon_{c n}$ beyond which there is no separatrix (see Figures 9 and 10 in [1]). It can be shown that trapping of low energy electrons occurs for $\epsilon$ values slightly lower that the critical one, $\epsilon \lesssim \epsilon_{c n}$. For $\epsilon=\epsilon_{c n}$ the adiabatic invariant takes the very simple expression $J_{s}=2 I_{r s}$ where $I_{r s}$ is the resonant action value, solution of the EC resonant condition $\partial H_{0} / \partial I=0$ that reads $\gamma-n \Omega / \omega-N_{\|} p_{z} / m c=0$ outside the beam region. Under the above conditions the nonlinear energy variation expe- 

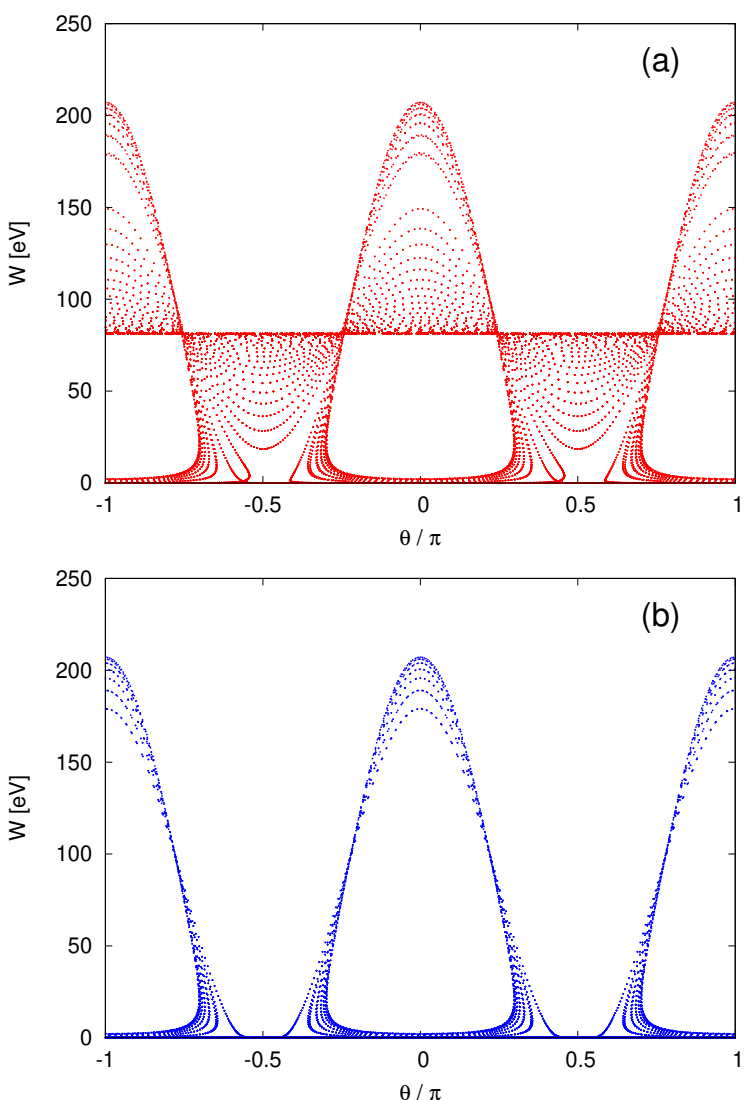

Figure 2. Same simulations as in Figure 1 in the "phase" space $(W, \theta)$.

rienced by cold electrons reads

$$
\Delta \gamma=2 v_{n}\left(I_{r s}-I_{0}\right) \approx 2 I_{r s},
$$

The energy gain is proportional to the distance from the resonant action in momentum space (at $\bar{P}_{z} \approx$ constant) and is maximum for particle with the lowest possible perpendicular energy $I_{0}$. This result is sketched in Figure 4 for the case of extraordinary mode in perpendicular propagation (see in [8] for a more detailed analysis). The lowest blue curve denotes the initial condition at low energy, the dashed black curve the EC resonant condition, and the red upper curve the final energy state after nonlinear interaction.

As it is clear from Eq.(8), the final energy state is independent of the electric field amplitude (i.e. of the beam power and width) for given ratio of the wave frequency to the cyclotron frequency and propagation angle. It is also independent of the detailed motion in the illumination region, provided trapping occurs. The overall process occurs with a given probability $q$, with $0 \leq q \leq 1$. The transition probability has been estimated numerically assuming that the initial particle distribution in parallel and perpendicular momentum is a Maxwellian function with temperature $T_{e}=0.03 \mathrm{eV}$, with initial phases $\theta$ distributed uniformly. The simulations have been performed for a large number of particles and the probability $q$ has been estimated as the fraction of particles undergoing a net variation for given $p_{\|}, p_{\perp}$. In Figure 5 a the result of a simulation is reported
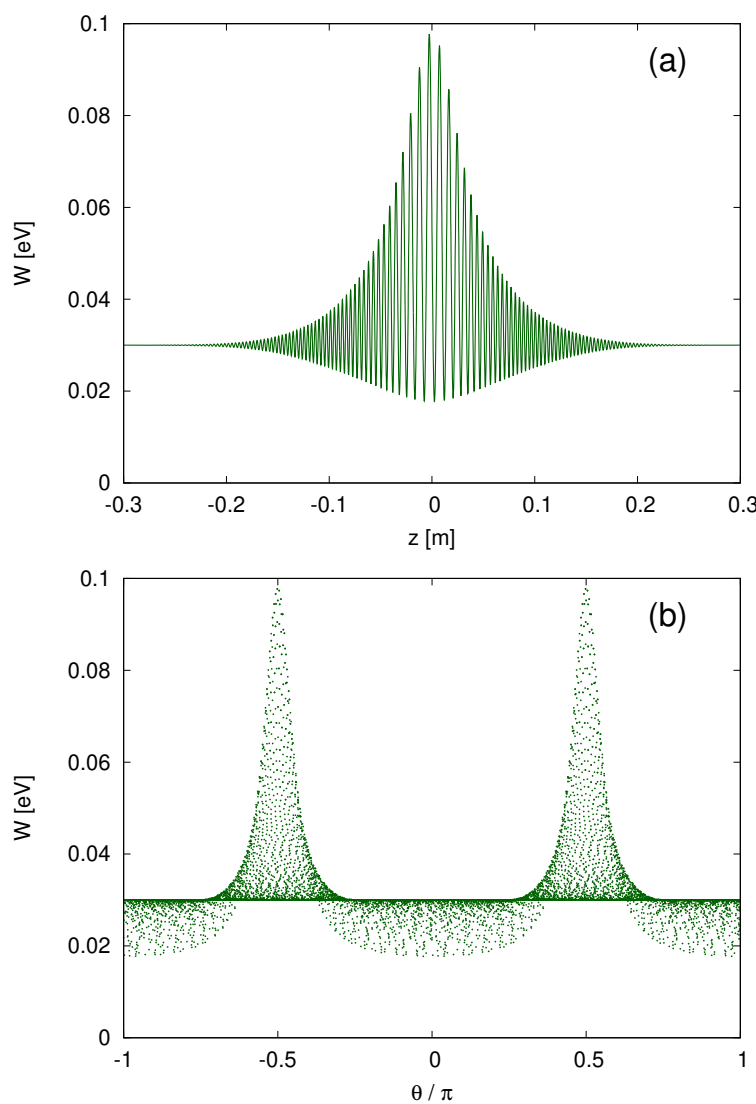

Figure 3. Example of motion for a non trapped particle in the $(W, z)$ plane ( (a)), and $(W, \theta)$ plane ( (b)). Same parameters as in Figure 1, at lower power $P_{E C}=300 \mathrm{~kW}$.

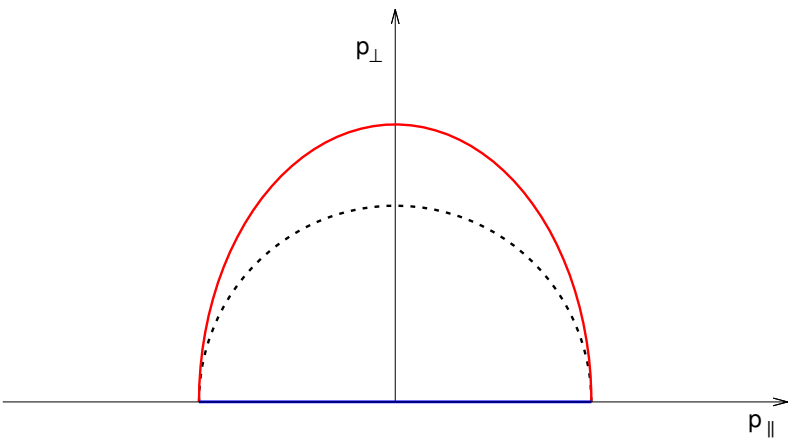

Figure 4. The energy variation process is sketched in the $\left(p_{\|}, p_{\perp}\right)$ plane for $\mathrm{XM}$ in perpendicular propagation. Initially the cold electrons lie on the blue line close to the axis origin. The dashed black curve is the resonant condition $\gamma=n \Omega / \omega$, and the red curve is the final energy given by Eq. (8).

in the momentum space for second harmonic extraordinary mode in perpendicular propagation and 48000 particles (i.e. $40 \times 40 \times 30$ for $p_{\perp}, p_{\|}$, and $\theta$ ). Note that the momentum is normalised to the initial thermal momentum. The resonant condition and the final energy are not plotted in the same scale, the resonant momentum being $\approx 58 p_{t h}$ and the final energy $\sim 2.5 \times 10^{3} T_{e}$. Probability $q$ is quite close to 1 for most initial conditions satisfying trapping condition (i.e., the dynamics of the majority of 

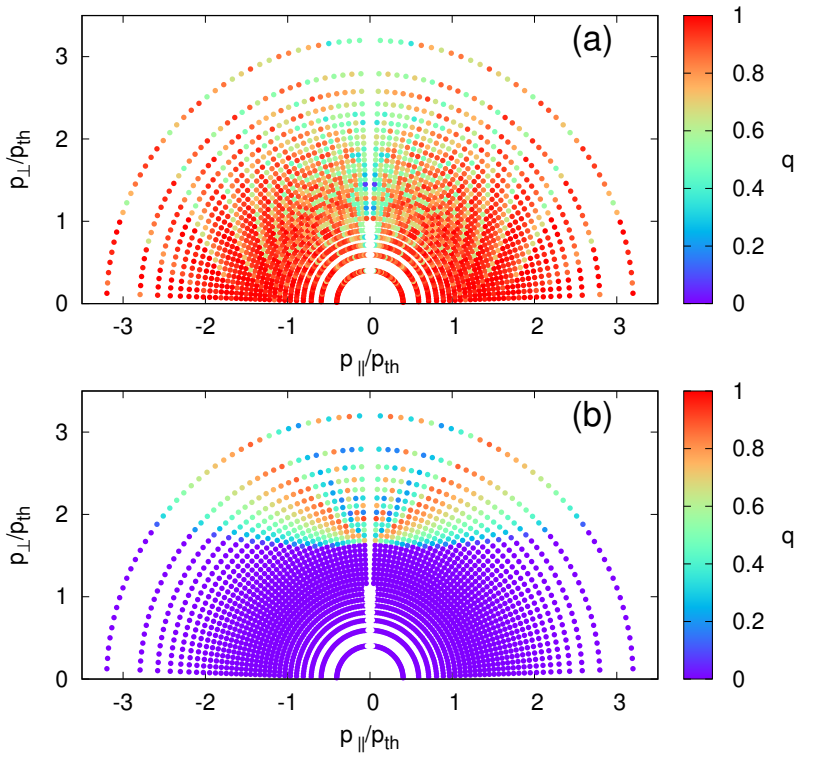

Figure 5. Probability transition $q$ to a large energy state reported in the momentum plane of initial conditions $p_{\| 0}, p_{\perp 0}$. For each couple of momentum values, the evolution of $N_{\theta}=30$ particles with uniformly distributed phases $\theta$ is followed through the EC illumination region. Probability $q$ is defined as the number of particles that experience the finite energy variation divided by $N_{\theta}$. XM2 perpendicular injection, with $P_{E C}=1 \mathrm{MW}$ and $1-\omega / 2 \Omega=$ $10^{-4}(\mathrm{a})$, and $1-\omega / 2 \Omega=1.22 \times 10^{-4}$ (b).

the particles is that illustrated in Figures 1a and 2a). If the frequency distance from the resonance is increased keeping the field amplitude fixed, the coldest particles are no more trapped and $q$ drops to zero in a large region of the momentum space as it is shown in Figure 5b. It should be noted that the understanding of the observed numerical results for the probability transition $q$ require an additional careful theoretical investigation which is beyond the scope of this paper.

\section{Energy gain}

The energy $W$ gained by an electron interacting with a localized EC beam is given by

$$
W=m c^{2} \Delta \gamma \cong 2 m c^{2} I_{r}\left(\bar{P}_{z s}\right)
$$

where the resonant action reads in the weakly relativistic approximation

$$
I_{r}\left(\bar{P}_{z}\right) \cong \frac{1-v_{n}+N_{\|} \bar{P}_{z}-\bar{P}_{z}^{2} / 2}{1-N_{\|}^{2}}
$$

The gained energy $W$ is a function of the frequency ratio $v_{n}$, of the parallel refractive index $N_{\|}$and of the parallel momentum at the separatrix crossing $\bar{P}_{z s}$. Note that the explicit expressions in Eqs. $(9,10)$ are valid in the limit $1-v_{n} \ll 1$ which is well verified for the pre-ionization parameters. As shown in [1], the variation of the canonical momentum $\bar{P}_{z}$ is negligible except for the first harmonic, oblique propagation, where it is finite although not large.
Thus, one can safely use the initial parallel canonical momentum $\bar{P}_{z 0}$ in Eq.(10), i.e. $I_{r s} \approx I_{r}\left(\bar{P}_{z 0}\right)$, except in the case $n=1, N_{\|} \neq 0$, where $I_{r s}$ can be estimated as

$$
I_{r s} \simeq \frac{1-v}{1-N_{\|}^{2} \mp N_{\|}\left[\left(1-N_{\|}^{2}\right) / 3\right]^{1 / 2}}
$$

in the limit $|1-v| \gg\left|\bar{P}_{z 0}\right|$. The two solutions correspond to particles passing and reflected by the EC nonlinear ponderomotive potential.

The energy $W$ increases linearly with the frequency ratio up to a critical value $\epsilon_{c n}$, where it achieves its maximum value beyond which it drops to zero. The critical frequency value corresponds to electrons crossing the separatrix for critical field value equal to the peak value $\epsilon_{M}=\left|q_{e}\right| E_{M} / m \omega$

$$
\epsilon_{M}=\epsilon_{c n} \equiv \beta_{c n} I_{r s}^{2-n / 2}\left(1-N_{\|}^{2}\right) / T_{n}
$$

where $\beta_{c 1}=(2 / 3)^{3 / 2}, \beta_{c 2}=1 / 2$ and the polarization term $T_{n}=N_{\perp}^{n-1}\left(e_{x}-e_{y}+e_{z} N_{\perp} \bar{P}_{z}\right) / 2$. The maximum energy gained at the critical frequency ratio $\omega / n \Omega$ is implicitly defined by Eq.(12) and reads

$$
W_{\text {max }}=2 m c^{2}\left(\frac{\epsilon_{M} T_{n}}{\beta_{c n}\left(1-N_{\|}^{2}\right)}\right)^{2 /(4-n)}
$$

For oblique propagation with finite $N_{\|}$the ordinary mode yields results similar to extraordinary mode, while energy values lower by the factor $\beta_{t h}^{2 /(4-n)}$ are found for perpendicular propagation.

\section{Spatial localisation}

\subsection{Radial profile}

As shown in Eqs.(9) and (10), at dominant order the gained energy $W$ depends linearly on the ratio between the wave frequency and the local cyclotron frequency $v_{n} \equiv \omega / n \Omega$. In the null region of a tokamak, the ratio $v_{n}$ is equal simply to the ratio between the local major radius $R$ and the radius of the cold cyclotron resonance $R_{E C n}$, defined by $\omega=n \Omega\left(R_{E C n}\right)$, so that the dependence of $W$ on the major radius can be obtained straighthforward.

Examples of the radial profile in ITER of the energy $W$ are shown in Figure 6 for XM2 and $N_{\|}=0$ for two values of the input power and in Figure 7 for XM2 at fixed power for two $N_{\|}$values. The analytical estimate derived in Sec. 3 is compared with the numerical result obtained solving the Hamiltonian equations of motion for a uniformly distributed set of initial phase values $\theta$ and same thermal initial action $I_{0}=\beta_{t h}^{2} / 2$ and $P_{z 0}=\beta_{t h}$. The agreement between the numerical simulations and the analytical estimates is extremely good, note that no free parameters are used here.

Making use of the relationship

$$
\epsilon_{M} \simeq 2.043 \times 10^{-3}\left(P / w^{2}\right)^{1 / 2} / f
$$

the maximum electron energy (13) can be expressed in terms of power $P$ in MW, frequency $f$ in $\mathrm{GHz}$ and beam 


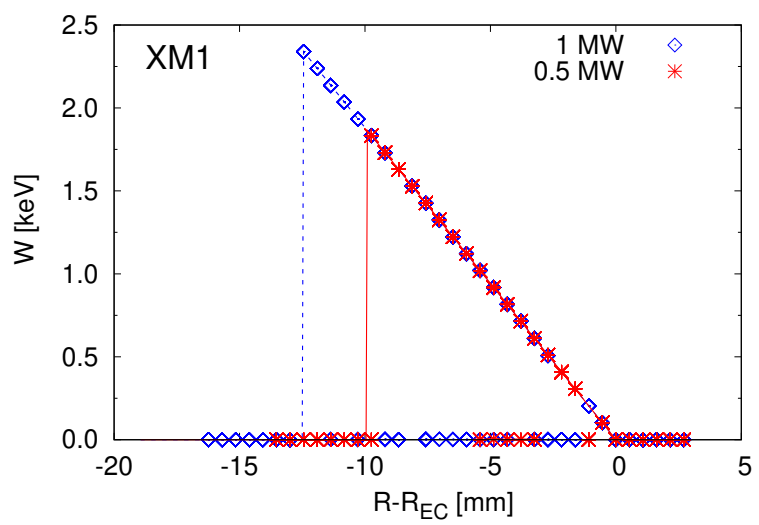

Figure 6. Radial energy profile for XM1 perpendicular propagation and $P_{E C}=0.5,1 \mathrm{MW}$ in ITER, with $R_{E C} \approx 5.41 \mathrm{~m}$ at full field $B_{0}=5.3 \mathrm{~T}$.

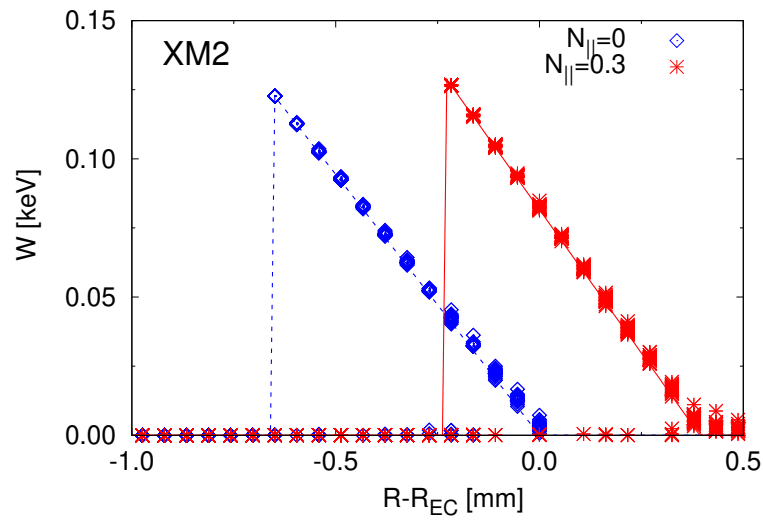

Figure 7. Radial energy profile for XM2 for $N_{\|}=0,0.3$ and $P_{E C}=1 \mathrm{MW}$ in ITER $\left(R_{E C 2}=R_{E C}\right.$ at half field $B_{0}=2.65 \mathrm{~T}$. $)$

spot sizes in $\mathrm{m}$. In case of XM in perpendicular propagation one obtains

$$
\begin{aligned}
& W_{\text {max }, n=1} \cong 15.55 P^{1 / 3} /(f w)^{2 / 3} \quad[\mathrm{keV}] \\
& W_{\text {max }, n=2} \cong 2.09 P^{1 / 2} /(f w) \quad[\mathrm{keV}] .
\end{aligned}
$$

The width of the radial region in which the energy gain process occurs is given by

$$
0<1-R / R_{E C n}<W_{\max , n} / 2 m c^{2} .
$$

The maximum electron energy Eq.(15) and the radial width of the interaction region Eq.(16) are shown in Figures 8 and 9 versus the local beam size for various input power in the ITER case for XM1 and XM2 in perpendicular propagation and injected frequency $170 \mathrm{GHz}$. Note that both the final energy and the radial width are much larger for first harmonic than for second harmonic (approximately by one order of magnitude for the ITER case).

\subsection{D Gaussian beam}

Let us consider a more realistic although simple injection configuration and a two dimensional EC beam, introduc-

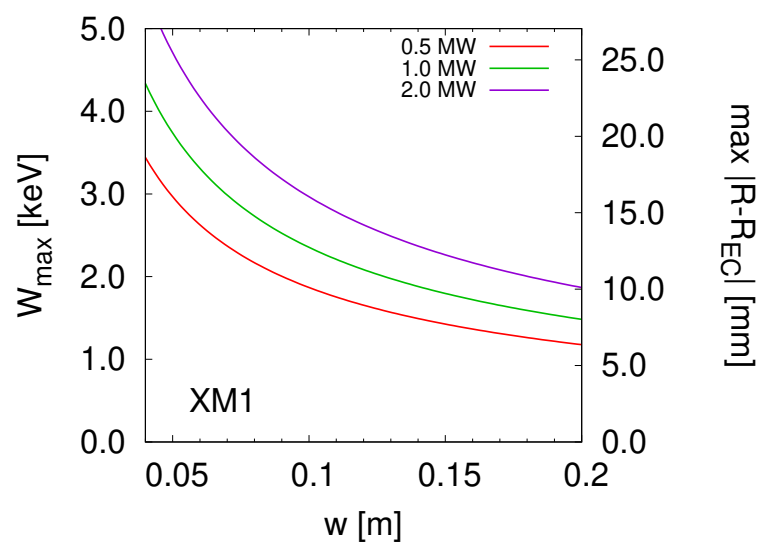

Figure 8. Maximum gained energy (left) and radial width of the interaction region (right) versus local beam width for $170 \mathrm{GHz}$, XM1 perpendicular propagation and various input power values.

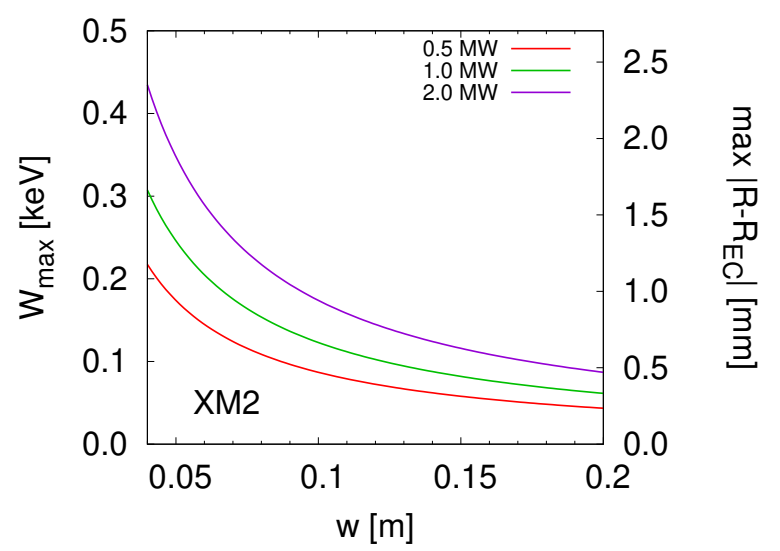

Figure 9. Same as in Figure 8 for XM2 in perpendicular propagation.

ing a rectified tokamak geometry with cartesian coordinate system $(x, y, z)$ related to the Tokamak system $(R, \phi, Z)$ as follows: $x=R-R_{E C}, y=Z$ and $z$ along $\phi$. For simplicity, hereafter we present results for the case of propagation perpendicular to the magnetic field $\mathbf{B}_{0}$, in the horizontal plane, i.e., $\mathbf{k}$ along $x$ axis.

The EC Gaussian beam is assumed to be astigmatic in the general case, with local beam width both in the $y$ and $z$ directions, and electric field amplitude given by

$$
E(y, z)=E_{M} g_{y}(y) g_{z}(z)
$$

with $g_{y}(y)=\exp \left(-y^{2} / w_{y}^{2}\right)$ and $g_{z}(z)=\exp \left(-z^{2} / w_{z}^{2}\right)$. On a given magnetic field line, i.e. at fixed $x$ and $y$ in this simplified geometry, an electron will experience a normalised e. m. amplitude variation $\epsilon=\epsilon_{M} g_{y}(y) g_{z}(z)$. The results derived in the previous sections are still valid applying the transformations $\epsilon_{M} \rightarrow \epsilon_{M} g_{y}(y)$, and $w^{2} \rightarrow w_{y} w_{z}$ [in Eq.(14)].

Both the maximum energy gain and the width of the radial interaction region exhibit a Gaussian dependence on the coordinate $y$ 


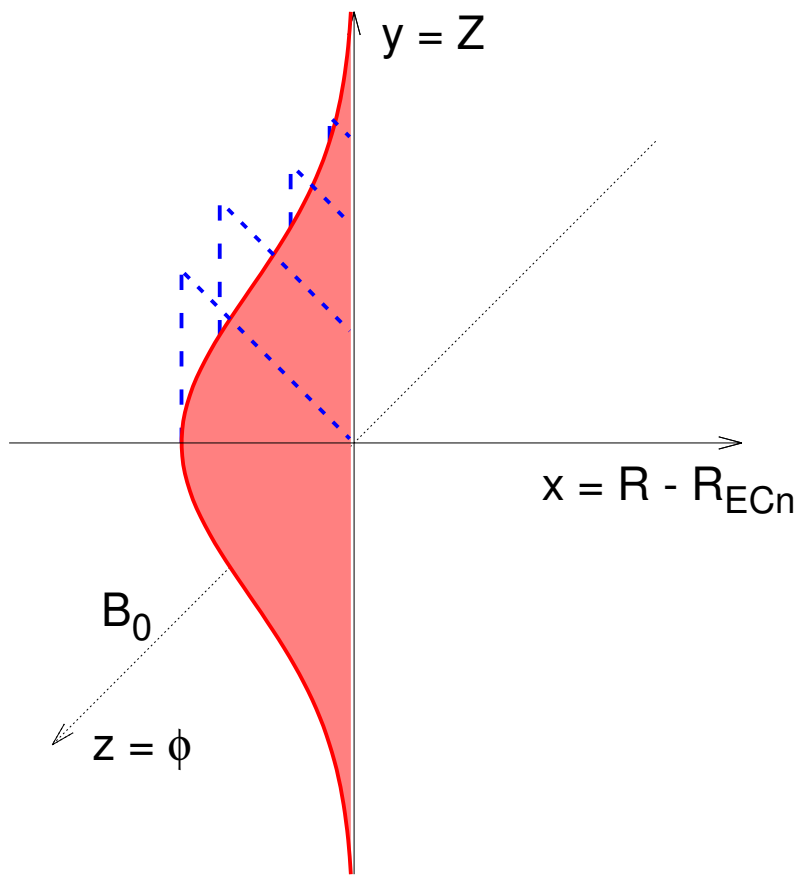

Figure 10. Region $\mathcal{R}_{x y}$ in $(x, y)$ plane where nonlinear EC interaction takes place (red filled region). Note that the picture is expanded in $x$ direction. The blue dashed lines are the energy profiles for different values of the $y$ coordinate.

$$
\begin{aligned}
& W_{\max , 1}(y)=3 m c^{2}\left[\epsilon_{M} g_{y}(y) / 2\right]^{2 / 3} \\
& W_{\max , 2}(y)=2 m c^{2} \epsilon_{M} g_{y}(y) .
\end{aligned}
$$

and

$$
\Delta R(y) \equiv\left|R_{E C n}-R\right|<R_{E C n} W_{\max , n}(y) / 2 m c^{2}
$$

A sketch of the region $\mathcal{R}_{x y}$ in the $x-y$ "poloidal" plane where condition (18) is verified is shown in Figure 10 (filled area). The dashed curves represent the energy spatial variation, with Gaussian shape $g_{y}(y)$. The picture is not realistic since it has been stretched in the $x$ direction, the $x$ scale being usually much smaller than the $y$ scale in experimental conditions, as it is clear from Eq. (18) and Figures 6, and 7.

An average energy value $\bar{W}_{n}$ can be introduced integrating $W$ over the region $\mathcal{R}_{x y}$ in the $x-y$ plane where condition (16) is satisfied (see Figure 10)

$$
\bar{W}_{n}=\frac{\iint d x d y W}{\iint d x d y}
$$

where the denominator represents the area $A_{n}$ of the interaction $\mathcal{R}_{x y}$. For a Gaussian amplitude profile it is found that

$$
\bar{W}_{n}=W_{\max , n}(y=0) / 2^{3 / 2} .
$$

which reads as a function of power $P$, frequency and beam spot sizes

$$
\begin{aligned}
& \bar{W}_{n=1} \simeq 5.50\left(P / w_{y} w_{z}\right)^{1 / 3} / f^{2 / 3}[\mathrm{keV}] \\
& \bar{W}_{n=2} \simeq 0.74\left(P / w_{y} w_{z}\right)^{1 / 2} / f[\mathrm{keV}]
\end{aligned}
$$

The area $A_{n}$ of the region $\mathcal{R}_{x y}$ (measured in $\mathrm{m}^{2}$ ) scales as

$$
\begin{aligned}
& A_{n=1} \simeq 3.3 \times 10^{-2} R_{E C}\left(P w_{y}^{2} / w_{z}\right)^{1 / 3} / f^{2 / 3} \\
& A_{n=2} \simeq 3.6 \times 10^{-3} R_{E C}\left(P w_{y} / w_{z}\right)^{1 / 2} / f .
\end{aligned}
$$

Note that both the average and the maximum energy depends on the beam spot sizes via their product.

For a uniform and constant density of seed electrons, the number of electrons $N$ acquiring a finite energy can be assumed to be proportional to the area $A_{x y}$ and to scale with beam local size as

$$
\begin{array}{lll}
N \propto\left(w_{y}^{2} / w_{z}\right)^{1 / 3} & \text { for } & n=1 \\
N \propto\left(w_{y} / w_{z}\right)^{1 / 2} & \text { for } & n=2
\end{array}
$$

\section{Conclusive remarks}

The analysis presented here provides analytical estimates which can give hints for the design of the experimental configuration of EC-assisted plasma breakdown. It is shown that electrons at room temperature can achieve finite energy via non linear collisionless wave-particle interaction. After being heated these electrons will collide with neutrals and ionise the gas and the plasma whenever their energy exceeds the ionization energy. Thus it is required that the gained energy is large enough to give rise to ionization (say $\sim 30 \mathrm{eV}$ ) and that a sufficient number of electrons are heated. This analysis is a first step towards a physics based approach to EC pre-ionization, and estimates (21) and (23) may give a practical contribution to its optimization.

This work was partially carried out within the framework of the ECHUL consortium, supported by the F4E grant F4E-GRT-615. The views and opinions expressed herein do not necessarily reflect those of the European Commission or the ITER Organisation.

\section{References}

[1] D. Farina, Nucl. Fusion, 58066012 (2018)

[2] D. Farina, EPJ Web Conf., 15703012 (2017)

[3] E. V. Suvorov, M. D. Tokman, Sov. J. Plasma Phys. 14557 (1988)

[4] W. M. Nevins, T. D. Rognlien and B I Cohen Phys. Rev. Lett. 5960 (1987)

[5] I. A. Kotelnikov and G. V. Stupakov Phys. Fluids B 2 881 (1990)

[6] I. A. Kotelnikov and G. V. Stupakov J. Plasma Phys. 4519 (1991)

[7] D. Farina, R. Pozzoli and D. Ronzio Plasma Phys. Control. Fusion 33485 (1991)

[8] D. Farina and R. Pozzoli Phys. Fluids B 31570 (1991)

[9] D. Farina, R. Pozzoli and M. Romé Phys. Fluids B 3 3065 (1991) 
